\title{
Excitation-energy influence at the scission configuration
}

\author{
D. Ramos ${ }^{1, \text { a }}$, C. Rodríguez-Tajes ${ }^{2}$, M. Caamaño ${ }^{1}$, F. Farget ${ }^{2}$, L. Audouin ${ }^{3}$, J. Benlliure ${ }^{1}$, E. Casarejos ${ }^{4}$, E. Clement ${ }^{2}$, \\ D. Cortina ${ }^{1}$, O. Delaune ${ }^{2, b}$, X. Derkx ${ }^{5}$, A. Dijon ${ }^{2}$, D. Doré ${ }^{6}$, B. Fernández-Domínguez ${ }^{1}$, G. de France ${ }^{2}$, A. Heinz $^{7}$,

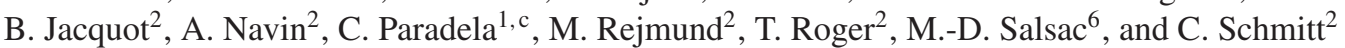 \\ 1 USC, E-15784 Santiago de Compostela, Spain \\ 2 GANIL, CEA/DMS-CNRS/IN2P3, BP. 55027, 14076 Caen Cedex 05, France \\ 3 IPN Orsay, Université de Paris-Sud, CNRS/IN2P3, 91406 Orsay Cedex, France \\ 4 CIMA, UVigo, 36310 Vigo, Spain \\ 5 LPC Caen, Université de Caen Basse-Normandie-ENSICAEN-CNRS/IN2P3, 14050 Caen Cedex, France \\ ${ }^{6}$ CEA Saclay, DMS/IRFU/SPhN, 91191 Gif-sur-Yvette Cedex, France \\ 7 Chalmes University of Technology, 41296 Göteborg, Sweden
}

\begin{abstract}
Transfer- and fusion-induced fission in inverse kinematics was proven to be a powerful tool to investigate nuclear fission, widening the information of the fission fragments and the access to unstable fissioning systems with respect to other experimental approaches. An experimental campaign for fission investigation has being carried out at GANIL with this technique since 2008. In these experiments, a beam of ${ }^{238} \mathrm{U}$, accelerated to $6.1 \mathrm{MeV} / \mathrm{u}$, impinges on a ${ }^{12} \mathrm{C}$ target. Fissioning systems from $\mathrm{U}$ to $\mathrm{Cf}$ are populated through transfer and fusion reactions, with excitation energies that range from few $\mathrm{MeV}$ up to $46 \mathrm{MeV}$. The use of inverse kinematics, the SPIDER telescope, and the VAMOS spectrometer permitted the characterization of the fissioning system in terms of mass, nuclear charge, and excitation energy, and the isotopic identification of the full fragment distribution. The neutron excess, the total neutron multiplicity, and the even-odd staggering in the nuclear charge of fission fragments are presented as a function of the excitation energy of the fissioning system. Structure effects are observed at $\mathrm{Z} \sim 50$ and $\mathrm{Z} \sim 55$, where their impact evolves with the excitation energy.
\end{abstract}

\section{Introduction}

The fission process is understood as a long and complex process involving extreme deformations, nuclear structure, and heat flows that decide the characteristics of the emerging fission-fragment distributions [1-4]. Nevertheless, despite almost 80 years of intense research, fission is still far from being fully understood, and the theoretical and experimental knowledge is not yet complete.

Nuclear fission at low excitation energy is one of the nuclear phenomena where both the macroscopic and microscopic aspects of the nuclear matter are involved: the deformation that the fissioning system reaches at the scission point corresponds to a collective movement of the nucleons, while the produced fragment distributions are strongly affected by the structure of their energy levels $[5,6]$.

Structure effects are revealed in the asymmetric component of the fission distributions which, in the actinides, was observed to remain rather constant around $\mathrm{A} \sim 140$ and $\mathrm{Z} \sim 54$, independent of the fissioning system $[7,8]$. However, only the recent use of the inverse kinematics technique coupled to a magnetic spectrometer permitted

\footnotetext{
a e-mail: diego.ramos@usc.es

${ }^{b}$ Present Address: CEA DAM DIF, 91297 Arpajon, France

${ }^{c}$ Present Address: EC-JRC-IRMM, Retieseweg 111, 2440 Geel, Belgium
}

the simultaneous measurement of both nuclear charge and mass distributions of fission fragments [9-12].

The present work provides a new ensemble of measurements that relates the structure effects that characterize the scission point, extracted from the identification of the full isotopic fission-fragment distributions, to the excitation energy of the fissioning system.

\section{Experimental setup}

Neutron-rich actinides are produced through transfer and fusion reactions with a beam of ${ }^{238} \mathrm{U}$ at $6.14 \mathrm{MeV} / \mathrm{u}$ impinging on a ${ }^{12} \mathrm{C}$ target. The actinides are identified through the target-like recoil ejected from the transfer reaction. This recoil is detected in the SPIDER telescope, composed of two double-sided, annular, Si detectors, where the isotopic identification of the recoil is achieved measuring both $\Delta E$ and $E$. The detector, divided in rings and sectors, provides also a measurement of both polar and azimuthal angles, permitting the reconstruction of the binary reaction, and thus, a measurement of the excitation energy, event by event, with an uncertainty of $\sim 1 \mathrm{MeV}$ [13]. A fusion reaction, ten times more frequent than any transfer channel, is assumed for every fission event that is registered without any target-like recoil detected in the telescope.

The fission decay of the actinides produces two fragments forward emitted within a cone of $25^{\circ}$. One of the 
fragments passes through the VAMOS spectrometer and it is fully identified in terms of mass, nuclear charge, and charge state [9]. The time-of-flight is measured from two multiwire chambers; both horizontal and vertical positions at the focal plane, as well as both angles are obtained through two drift chambers; the energy loss is measured in an ionization chamber; and the remaining energy, in a wall of silicon detectors. The angles at the target position and the magnetic rigidity of the fission fragments are reconstructed by back-tracing methods based on the ionic optics of the spectrometer $[14,15]$. The acceptance of the spectrometer is corrected for each pair of mass and charge state of the fission fragments as shown in [9].

Finally, six clovers of the EXOGAM array of Ge detectors were placed surrounding the target for $\gamma$-ray measurements, yielding information on the sharing of the excitation energy between the fissioning system and the target-like recoil [13], and providing a verification of the fragment identification [16].

\section{Fission products}

The fission products of five fissioning systems are reported in this work: ${ }^{238} \mathrm{U},{ }^{239} \mathrm{~Np},{ }^{240} \mathrm{Pu},{ }^{244} \mathrm{Cm}$, produced through transfer reactions with distributions of excitation energy centered at $\left\langle E_{x}\right\rangle=7.4 \mathrm{MeV},\left\langle E_{x}\right\rangle=7.5 \mathrm{MeV},\left\langle E_{x}\right\rangle=$ 10.7 MeV, and $\left\langle E_{x}\right\rangle=23.0 \mathrm{MeV}$ respectively, with a full width at half maximum of approximately $8 \mathrm{MeV}$, and ${ }^{250} \mathrm{Cf}$, produced through fusion reactions with an excitation energy of $E_{x}=46 \mathrm{MeV}$. A detailed description of the $E_{x}$ distribution of each system is presented in [13].

The measurement of isotopic fission yields achieved in this work [17] permits to investigate the fission process in terms of the neutron and proton contents of fission fragments after post-scission evaporation through the neutron excess and the total neutron multiplicity, while the elemental yields provide information about the rupture of pairs from the saddle to the scission point through the evenodd staggering in $\mathrm{Z}$.

\subsection{Neutron excess}

In order to study the sharing of protons and neutrons, the neutron excess is defined as the average number of neutrons per proton in the fragments,

$$
\frac{\langle N\rangle}{Z}(Z)=\frac{1}{Z} \sum_{N} N \frac{Y(N, Z)}{Y(Z)},
$$

where $Y(N, Z)$ and $Y(Z)$ represent the isotopic and elemental yields.

Figure 1 presents the neutron excess of the five fissioning systems as a function of the fragment nuclear charge. The systems at lower $E_{x}$ show a strong charge polarization with a maximum at $\mathrm{Z} \sim 50$ that breaks the overall increasing trend observed in the systems at higher $E_{x}$. The doubly-magic nucleus ${ }^{132} \mathrm{Sn}$, indicated with a black dot, is understood to be the promoter of such large neutron excess. The effect of the deformed shell $\mathrm{N} \sim 88$, indicated with a dashed line, is partially reduced due to the neutron evaporation.

The evolution of the neutron excess of ${ }^{240} \mathrm{Pu}$ as a function of $E_{x}$ is presented in Fig. 2. $E_{x}$ reduces the neutron excess in the heavy fragment in a systematic

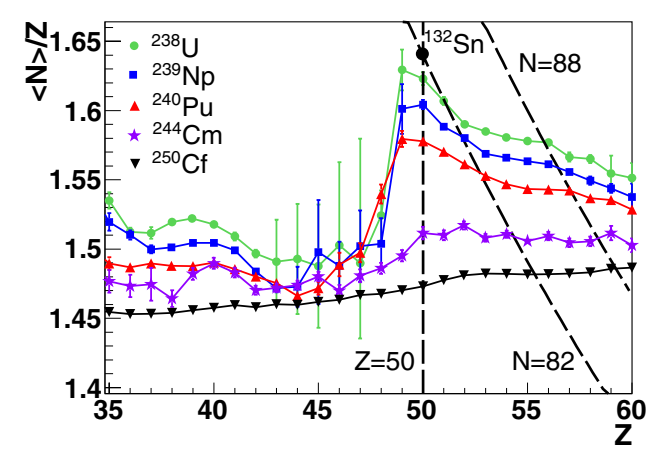

Figure 1. Comparison of the neutron excess as a function of the fragment nuclear charge of the five fissioning systems discussed in this work, with the $\left\langle E_{x}\right\rangle$ presented at the beginning of the Sect. 3. The doubly-magic nucleus ${ }^{132} \mathrm{Sn}$ and the spherical shell $\mathrm{Z}=50$ and $\mathrm{N}=82$, as well as the deformed shell $\mathrm{N} \sim 88$, are indicated in the picture.

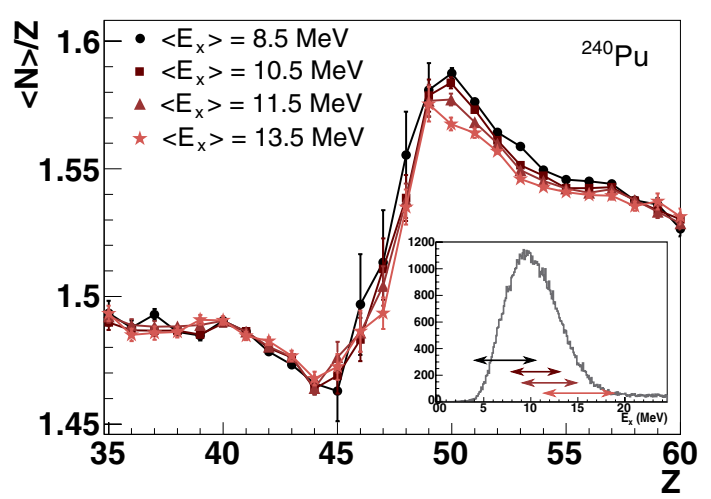

Figure 2. Neutron excess as a function of the fragment nuclear charge of ${ }^{240} \mathrm{Pu}$ for different $E_{x}$. The inset presents the full $E_{x}$ distribution and the ranges taken for each set of data.

way larger than the effect of the uncertainty, and with a larger influence around $\mathrm{Z} \sim 50$, while the light fragment remains almost unaffected. This effect is coherent with the evolution of the neutron multiplicity with $E_{x}$ observed in [18], where the excess of $E_{x}$ produced larger evaporation in the heavy fragment.

\subsection{Total neutron multiplicity}

The total neutron multiplicity $\left(\langle v\rangle_{\text {total }}\right)$ is obtained, as a function of the fragment nuclear charge, from the isotopic fragment yields as the difference between the number of neutrons of the fissioning system $\left(N_{\text {fiss }}\right)$ and the sum of the average number of neutrons of both complementary fission fragments,

$$
\langle v\rangle_{\text {total }}(Z)=N_{\text {fiss }}-\left(\left.\langle N\rangle\right|_{Z}+\left.\langle N\rangle\right|_{Z_{\text {fiss }}-Z}\right) .
$$

Figure 3 shows the total neutron multiplicity of the five fissioning systems as a function of the fragment nuclear charge. The neutron multiplicity increases for systems at higher $E_{x}$, with a maximum for symmetric splits, indicating larger deformation at scission. A minimum multiplicity appears around $\mathrm{Z} \sim 50$ in systems at low $E_{x}$, suggesting the effect of the spherical shells $Z=50$ and $\mathrm{N}=82$. A local maximum is observed around $\mathrm{Z} \sim 55$, also present in ${ }^{250} \mathrm{Cf}$ shown in the inset, which is coherent with the large deformation of the shell $\mathrm{N} \sim 88$. 


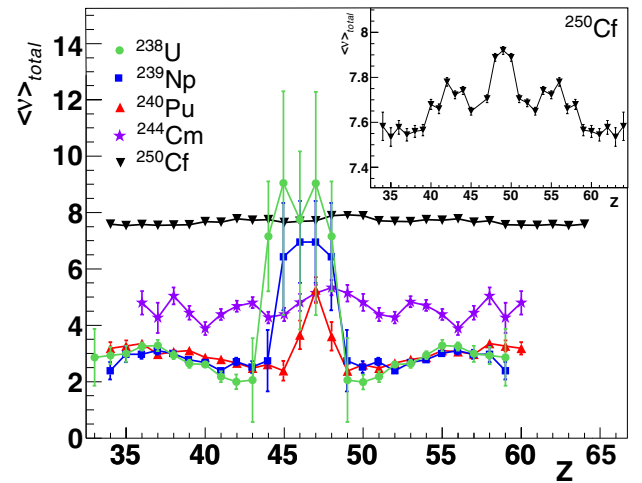

Figure 3. Total neutron multiplicity as a function of the fragment nuclear charge. The $\left\langle E_{x}\right\rangle$ of each system corresponds to the values presented at the beginning of the Sect. 3 . The inset presents a magnified view of the ${ }^{250} \mathrm{Cf}$ system.

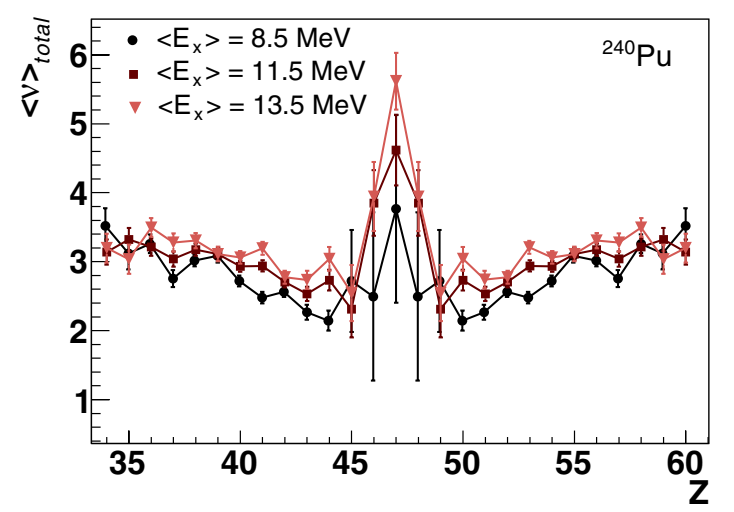

Figure 4. Total neutron multiplicity of ${ }^{240} \mathrm{Pu}$ as a function of the fragment nuclear charge for different $E_{x}$.

Figure 4 presents the total neutron multiplicity as a function of the fragment nuclear charge in ${ }^{240} \mathrm{Pu}$ for different $E_{x}$. The minimum around $\mathrm{Z} \sim 50$ disappears with increasing $E_{x}$, while the split $\left(Z_{1}, Z_{2}\right)=(39,55)$ remains unaffected in the range of $E_{x}$ investigated. The particular strong evolution around $\mathrm{Z} \sim 50$ is coherent with the presence of shell effects: at low $E_{x}$, a heavy fragment around $Z \sim 50$, also affected by the spherical shell $\mathrm{N}=82$, has a lower neutron evaporation probability that its neighbors, and this difference reduces at higher $E_{x}$ since the shell effect vanishes with $E_{x}$. The unaffected pair $\left(Z_{1}\right.$, $\left.Z_{2}\right)=(39,55)$ suggests an effect of a deformed shell that blocks a specific deformation.

\section{3. $Z$ even-odd staggering}

For low energy fission, experimental data show that even fragments are systematically more produced than odd fragments. This effect is a consequence of the pairing energy: the formation of odd-odd fragments needs higher energy in order to break nucleon pairs than the formation of even-even fragments [19].

The global even-odd staggering in nuclear charge is calculated in the five fissioning systems investigated in this work as:

$$
\delta_{\text {global }}=\frac{1}{\sum_{Z} Y(Z)} \sum_{Z}(-1)^{Z} Y(Z),
$$

where $Y(Z)$ are the elemental yields.

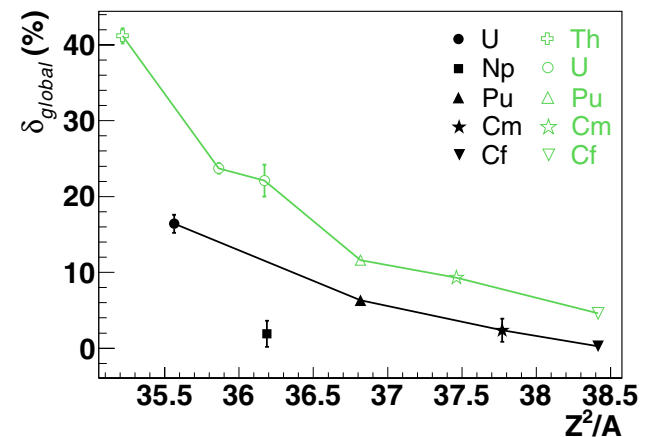

Figure 5. Global even-odd staggering as a function of the fissility parameter. Present data (black) are compared with thermalneutron induced fission (green), extracted from [20].

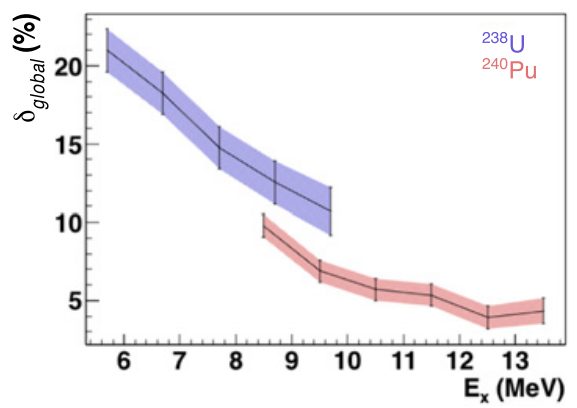

Figure 6. Global even-odd effect as a function of $E_{x}$ in ${ }^{238} \mathrm{U}$ and ${ }^{240} \mathrm{Pu}$.

Figure 5 presents the global even-odd staggering in nuclear charge as a function of the fissility parameter $\left(Z^{2} / A\right)$, which relates the mass and the nuclear charge of the fissioning system. Present data (black) are compared with $\left(n_{t h}, f\right)$ data (green) [20]. Even-Z systems follow a decreasing behavior with $Z^{2} / A$ with a shift between present data and $\left(n_{t h}, f\right)$ data produced by the difference in $E_{x}$ : a system with an excitation energy $E_{x}$ at the ground state, reaches the saddle point with intrinsic excitation energy $\left(E_{x}-B_{f}\right)$, being $B_{f}$ the fission barrier. This intrinsic energy adds to the energy dissipated form saddle to scission, thus, the higher the excitation energy above the barrier, the lower the even-odd effect. The odd-Z system ${ }^{239} \mathrm{~Np}$ presents lower $\delta_{\text {global }}$ because of the continuous presence of at least one unpaired proton.

The global even-odd staggering in nuclear charge as a function of $E_{x}$, presented in Fig. 6 for ${ }^{238} \mathrm{U}$ and ${ }^{240} \mathrm{Pu}$, shows a decreasing exponential behavior produced by the saturation of the pair-rupture at high $E_{x}$. The shift between both systems reflects the different asymmetry of the systems [20].

The local even-odd staggering in nuclear charge, calculated through the equation developed by B.L. Tracy et al. [21]:

$$
\begin{aligned}
\delta\left(Z+\frac{3}{2}\right)= & \frac{(-1)^{Z}}{8}[\ln Y(Z+3)-\ln Y(Z) \\
& -3(\ln Y(Z+2)-\ln Y(Z+1))],
\end{aligned}
$$

is investigated as a function of the asymmetry parameter:

$$
a(Z)=\frac{Z-\left(Z_{\text {fiss }}-Z\right)}{Z_{\text {fiss }}} .
$$




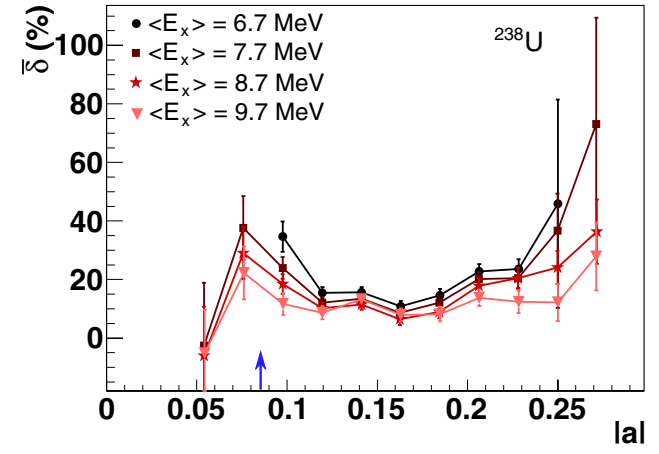

Figure 7. Local nuclear-charge even-odd staggering as a function of the absolute value of the asymmetry parameter for different $E_{x}$ in ${ }^{238} \mathrm{U}$. The blue arrow indicates the position of $\mathrm{Z}=50$.

Figure 7 presents the local nuclear-charge even-odd staggering of ${ }^{238} \mathrm{U}$ as a function of the absolute value of the asymmetry parameter for different $E_{x} . \delta$ is observed to be higher for higher asymmetry with a local maximum around $\mathrm{Z} \sim 50$, indicated with the blue arrow, reflecting the effect of the large binding energy compared with the neighbors. An increase in $E_{x}$ decreases the local even-odd staggering with a stronger evolution at high asymmetry and $\mathrm{Z} \sim 50$.

\section{Conclusions and outlook}

The fission process was experimentally investigated in n-rich actinides ${ }^{250} \mathrm{Cf},{ }^{244} \mathrm{Cm},{ }^{240} \mathrm{Pu}$, and ${ }^{239} \mathrm{~Np}$, and in ${ }^{238} \mathrm{U}$, produced through fusion and transfer reactions. The use of the inverse kinematics technique with the spectrometer VAMOS and the telescope SPIDER made the characterization of the fissioning system possible in terms of $\mathrm{Z}, \mathrm{A}$, and $E_{x}$, and the isotopic identification of the full fragment distributions.

The correlation between fission-fragment $\mathrm{N}$ and $\mathrm{Z}$ reveals the effect of structure: a charge polarization with a maximum around $\mathrm{Z} \sim 50$ governed by ${ }^{132} \mathrm{Sn}$. This structure effect reduces for increasing $E_{x}$, but mainly on the heavy fragment.

The signatures of structure effects on the total neutron multiplicity are a minimum corresponding to
$\mathrm{Z}=50$ spherical shell and a local maximum at $\mathrm{Z} \sim 55$, probably related with the $\mathrm{N} \sim 88$ deformed shell. The large evaporation at symmetry indicates large deformation at scission.

The global even-odd effect in even- $Z$ systems exhibits an exponential behavior with $E_{x}$. The local even-odd effect is found to increase around $\mathrm{Z} \sim 50$ splits, consistent with a larger binding energy, and at high asymmetry.

In summary, these measurements confirm the presence of structure effects and allow to characterize their dependence on $E_{x}$.

\section{References}

[1] J.L. Sida et al., Nucl. Phys. A 502, 233c (1989)

[2] S. Steinhäuser et al., Nucl. Phys. A 634, 89 (1998)

[3] C. Böckstiegel et al., Nucl. Phys. A 802, 12 (2008)

[4] K.-H. Schmidt and B. Jurado, Phys. Rev. Lett. 104, 212501 (2010)

[5] V.M. Strutinksy, Nucl. Phys. A 95, 420 (1972)

[6] B.D. Wilkins et al., Phys. Rev. C 14, 1832 (1976)

[7] K.F. Flynn et al., Phys. Rev. C 5, 1725 (1972)

[8] K.-H. Schmidt et al., Nucl. Phys. A 665, 221 (2000)

[9] M. Caamaño et al., Phys. Rev. C 88, 024605 (2013)

[10] M. Caamaño et al., Phys. Rev. C 92, 034606 (2015)

[11] J.-L. Rodríguez-Sánchez et al., Phys. Rev. C 91, 064616 (2015)

[12] J.-F. Martin et al., Eur. Phys. J. A 91, 174 (2015)

[13] C. Rodríguez-Tajes et al., Phys. Rev. C 89, 024614 (2014)

[14] S. Pullanhiotan et al., Nucl. Instr. and Methods A 593, 343 (2008)

[15] M. Rejmund et al., Nucl. Instr. and Methods A 646, 184 (2011)

[16] A. Shrivastava et al., Phys. Rev. C 80, 051305 (2009)

[17] D. Ramos et al., EPJ Web of Conferences 111, 10001 (2016)

[18] R. Müller et al., Phys. Rev. C 29, 885 (1984)

[19] F. Rejmund et al., Nucl. Phys. A 678, 215 (2000)

[20] M. Caamaño et al., J. Phys. G: Nucl. Part. Phys. 38, 035101 (2011)

[21] B.L. Tracy et al., Phys. Rev. C 5, 222 (1972) 